\title{
Prediction of mediastinal lymph node metastasis in adenocarcinoma of the esophagogastric junction
}

\author{
Henrik Nienhüser, Thomas Schmidt
}

Department of General, Visceral and Transplant Surgery, University of Heidelberg, Heidelberg, Germany

Correspondence to: Thomas Schmidt, MD, PhD. Department of General, Visceral \& Transplant Surgery, University of Heidelberg, Im Neuenheimer Feld 110, 69120 Heidelberg, Germany. Email: Thomas.Schmidt1@med.uni-heidelberg.de.

Provenance: This is an invited article commissioned by the Academic Editor Shuang-Jiang Li (Department of Thoracic Surgery and West China Medical Center, West China Hospital, Sichuan University, Chengdu, China).

Comment on: Mitchell KG, Ikoma N, Nelson DB, et al. Mediastinal Nodal Involvement After Neoadjuvant Chemoradiation for Siewert II/III Adenocarcinoma. Ann Thorac Surg 2019;108:845-51.

Submitted Sep 01, 2019. Accepted for publication Sep 29, 2019.

doi: $10.21037 /$ jtd.2019.10.28

View this article at: http://dx.doi.org/10.21037/jtd.2019.10.28

The optimal surgical management of adenocarcinoma of the esophagogastric junction (AEG) is challenging and complex. While Siewert type I tumors are treated according to the algorithm of esophageal cancer the optimal approach for Siewert II/III tumors is still under debate (1). In particular, the best operative approach and the extend of lymph node dissection were investigated in several studies without clear consensus $(2,3)$.

In The Annals of Thoracic Surgery, Kyle Mitchell and colleagues report their findings from a single-center study investigating predictive factors for mediastinal involvement in neoadjuvant treated patients with AEG II/ III tumors. Analyzing 204 patients they find that rather the pretreatment tumor extension into the esophagus than the Siewert classification is an independent predictor of mediastinal lymph node involvement. The authors calculate a best cut-off value of a proximal tumor extend of more than $15 \mathrm{~mm}$ as discriminative and suggest to use this parameter for the operative planning of patients with AEG II/III tumors.

The association between proximal tumor extend and lymph node involvement has been described by other authors in the past (4) but in the current study only patients who received trimodality therapy were included. Since the introduction of neoadjuvant therapy different techniques of preoperative staging to detect metastatic lymph nodes have been investigated. Because of the low accuracy of CT imaging position emission tomography (PET) has been applied to detect suspicious lymph nodes. While this method has a high specificity of more than $95 \%$ the sensitivity is only about $67 \%$ (5) and thereby reducing the diagnostic value for treatment decisions (6).

The study by Mitchell et al. has its major limitation at this point. The authors report a retrospective cohort of patients and state that staging included PET and endoscopic ultrasound with fine-needle aspiration (EUS/ FNA) according with the guidelines in effect at the time of treatment. It remains unclear how many patients underwent which preoperative work-up and this limits the comparability of the data. Because the operating surgeon made the decision of the approach individually for each patient, the authors do not have pathological data for patients who did not undergo a mediastinal lymphadenectomy. To compensate this, they define histopathological tumor evidence in mediastinal lymph nodes or a mediastinal recurrence within two years after the resection as mediastinal lymph node positive. This composite endpoint is on the one hand an elegant way in comparing the two different operative approaches but on the other hand carries a lot of potential bias. It would be interesting to know how many of these patients showed localized mediastinal recurrence and how many patients were diagnosed with mediastinal lymph node recurrence as part of a multifocal recurrence. Additionally the quality of the thoracic lymph node dissection is not described in detail. Different studies have shown that the number 
of resected lymph nodes correlates with the accuracy of staging and survival (7) and that a transthoracic approach leads to a higher number of resected lymph nodes (8). To get a better idea, the extend of lymph node dissection with the number of removed lymph nodes in each group would be very informative for this study.

The question if patients with Siewert II tumors should undergo a thoracic lymphadenectomy is still controversial and the current study adds more evidence for the clinical decision-making. Over ten years ago, Sasako et al. reported no benefit of a transthoracic approach for patients with cardia or subcardia cancer (9). At the same time, Omloo et al. published the first randomized controlled trial with longterm results on this question (10). This study did not show a survival benefit by a transthoracic approach in patients with AEG II in general, but was suggesting a benefit for patients with a high number of positive lymph nodes. Since publication of this study, several groups have shown that patients with AEG II tumors could benefit from an extended transthoracic lymphadenectomy but all studies are limited because of the small subgroup of patients with AEG II tumors (2). A recently registered international randomized controlled trial (CARDIA Trial DRKS00016923) will start recruitment this year and will generate high evidence data for the best operative approach in patients with AEG II tumors.

In the last years different authors have reported that not only the tumor type according to the Siewert classification, but also the tumor extend into the esophagus is an independent prognostic factor of mediastinal lymph node involvement and survival. Koyanagi et al. describe an invasion of more than $25 \mathrm{~mm}$ above the GEJ as independent risk factor for mediastinal involvement (11). For the patient cohort published in the current study Mitchell et al. calculate a best cut-off value of $15 \mathrm{~mm}$ above the GEJ as a predictive marker but report even patients with a lower tumor extend at risk for mediastinal metastasis. While the authors focus on the mediastinal lymph node involvement, they do not discuss the problem of distal tumor extend and abdominal lymphadenectomy. In a similar pattern to the risk of mediastinal lymph node metastasis, other groups have investigated the correlation between the distal tumor extend and abdominal lymph node metastasis in Siewert type II tumors. Mine et al. recommend an abdominal lymphadenectomy if the distal tumor extend is more than $30 \mathrm{~mm}$ from the GEJ (12).

In summary, the current study by Mitchell et al. provides surgeons with additional evidence for planning the operative approach in patients with Siewert II/III tumors. If the distance of $15 \mathrm{~mm}$ should be seen as a sharp cut-off value is even questioned by the authors themselves and it therefore provides another piece of information for our decision about the operative approach. Future studies like the above-mentioned CARDIA trial will hopefully shed additional light into this question.

\section{Acknowledgments}

None.

\section{Footnote}

Conflicts of Interest: The authors have no conflicts of interest to declare.

Ethical Statement: The authors are accountable for all aspects of the work in ensuring that questions related to the accuracy or integrity of any part of the work are appropriately investigated and resolved.

\section{References}

1. Gertler R, Stein HJ, Loos M, et al. How to classify adenocarcinomas of the esophagogastric junction: as esophageal or gastric cancer? Am J Surg Pathol 2011;35:1512-22.

2. Heger P, Blank S, Goossen K, et al. Thoracoabdominal versus transhiatal surgical approaches for adenocarcinoma of the esophagogastric junction-a systematic review and meta-analysis. Langenbecks Arch Surg 2019;404:103-13.

3. Blank S, Schmidt T, Heger P, et al. Surgical strategies in true adenocarcinoma of the esophagogastric junction (AEG II): thoracoabdominal or abdominal approach? Gastric Cancer 2018;21:303-14.

4. Meier I, Merkel S, Papadopoulos T, et al. Adenocarcinoma of the esophagogastric junction: the pattern of metastatic lymph node dissemination as a rationale for elective lymphatic target volume definition. Int J Radiat Oncol Biol Phys 2008;70:1408-17.

5. Ott K, Weber W, Siewert JR. The importance of PET in the diagnosis and response evaluation of esophageal cancer. Dis Esophagus 2006;19:433-42.

6. Schmidt T, Lordick F, Herrmann K, et al. Value of functional imaging by PET in esophageal cancer. J Natl Compr Canc Netw 2015;13:239-47.

7. Altorki NK, Zhou XK, Stiles B, et al. Total number of 
resected lymph nodes predicts survival in esophageal cancer. Ann Surg 2008;248:221-6.

8. Kutup A, Nentwich MF, Bollschweiler E, et al. What should be the gold standard for the surgical component in the treatment of locally advanced esophageal cancer: transthoracic versus transhiatal esophagectomy. Ann Surg 2014;260:1016-22.

9. Sasako M, Sano T, Yamamoto S, et al. Left thoracoabdominal approach versus abdominal-transhiatal approach for gastric cancer of the cardia or subcardia: a randomised controlled trial. Lancet Oncol 2006;7:644-51.

10. Omloo JM, Lagarde SM, Hulscher JB, et al. Extended transthoracic resection compared with limited transhiatal

Cite this article as: Nienhüser H, Schmidt T. Prediction of mediastinal lymph node metastasis in adenocarcinoma of the esophagogastric junction. J Thorac Dis 2019;11(11):E214-E216. doi: $10.21037 /$ jtd.2019.10.28 resection for adenocarcinoma of the mid/distal esophagus: five-year survival of a randomized clinical trial. Ann Surg 2007;246:992-1000; discussion 1000-1.

11. Koyanagi K, Kato F, Kanamori J, et al. Clinical significance of esophageal invasion length for the prediction of mediastinal lymph node metastasis in Siewert type II adenocarcinoma: A retrospective single-institution study. Ann Gastroenterol Surg 2018;2:187-96.

12. Mine S, Kurokawa Y, Takeuchi H, et al. Distribution of involved abdominal lymph nodes is correlated with the distance from the esophagogastric junction to the distal end of the tumor in Siewert type II tumors. Eur J Surg Oncol 2015;41:1348-53. 